\title{
Animal bone growth experiment of rapid-growing rats in different stress environment and its mathematical model ${ }^{*}$
}

\author{
Jun Zhang', Wenzhi Zhao ${ }^{2}$ \\ ${ }^{1}$ Advanced Technology of Transportation Vehicle Key Laboratory of Liaoning Province, Dalian Jiaotong University, Dalian, China \\ ${ }^{2}$ Department of Orthopaedics, The Second Affiliated Hospital of Dalian Medical University, Dalian, China \\ Email: armyzhang@sina.com
}

Received 2013

\begin{abstract}
The aim of this study is to explore a way that quantify the qualitative equation of bone growth and remodeling which was based on the animal Experiment of rapid-growing Rats in Different Stress Environment. These results were proved to be of good stability and identification precision with the numerical method of inversion. It suggested that the growing coefficient and the threshold in function were variables changing with time and space. The idea and method used in the research of bone growth and remodeling adaptation in this paper also provided clue and reference to establish other models for living system.
\end{abstract}

Keywords: Bone Growth Equation; Parameter Identification; Animal Experiment; Bone Mineral Density

\section{INTRODUCTION}

In the 21st century, bioengineering research, which is integrated with biology, mathematics, mechanics and computing science, is gradually to be a hot spot. Since last century, many researchers have been working in this field on biological experiments and theoretical deduction [1]. So far, few quantificational research findings about the life process were put forward because of the difficulty of combination of experimental results and theoretical studies. In this paper, biological parameters of bone growth equation were identified using the inversion theory by the computing numerical method. The investigation was based on the combination of animal experiment and its mathematical representation and provided chance of deeply discussing the relationship between life phenomenon and environmental stimulation.

In this study, on the basis of biological experiment on rapid growing rats, the effects of overloading and unloading on bone growth and remodeling were investi-

\footnotetext{
"Project of Liaoning Province Education Department, LS2010030.
}

gated. Different stress environments were created so that the rats' left femurs beared dissimilar stress stimulus from right femurs. The numerical value was obtained of proximal femur bone mineral density (BMD) at equal interval time within two months. The loading of each femur was calculated by the combination of the BMD value and the same rat individual body weight data. Based on the identification theory [2], we designed computer program and calculated the unknown coefficients in bone growth equation in different periods and moreover, the changing trend of rat femur BMD several weeks in the future was predicted.

\section{METHOD OF ANIMAL EXPERIMENTS}

After the "law of bone transformation [3]" put forwarded by Wolff in 1892, researchers characterized this theory as bone self-optimization process with mathematical method (as shown in Eq.1). In order to identify the unknown parameters in the equation, we carried out two experiments on rats within two years and got the data of bone mineral density (BMD) and load. The differences between the two experiments on the aspects of rats' selection, experiment instruments, data acquisition and analysis were controlled to be a minimum.

\subsection{Animal Selection, Grouping and Feeding}

60 six-week-old female Sprague Dawley rats (body mass $160 \pm 12 \mathrm{~g}$ ) obtained from the experimental animal center of Dalian Medical University were randomly divided into two groups: 45 animals as experiment group (E), the other 15 animals without any treatment were served as comparison group (C); In the experiment group, the animals were performed sciatic nerve resection of right hindlimb through a small incision in the gluteal area, under anesthesia (mebubarbital $30 \mathrm{mg} / \mathrm{kg}$ intraperitoneally injected). The right femurs which lost the ability of load were defined as unloading group (U) of experiment group and the left should bear more loads defined as overloading group $(\mathrm{O})$ of experiment group. 
After the operation, the rats were housed for 8 weeks individually in temperature-controlled room $\left(220^{\circ} \mathrm{C} \pm\right.$ $20^{\circ} \mathrm{C}$ ) with a $12: 12$ hour light/dark cycle. The animals have free access to water and a standard rat laboratory diet and were encouraged to move for 30 minutes in the morning, noon and evening respectively.

\subsection{Bone Mineral Density Data Obtaining and Analysis}

After the animals had been anesthetized and weighed, the bone mineral density(BMD) of rat femur was measured once a week, the value of area BMD of the same sit in the proximal Femur was obtained (as shown in Figure 1). The adopted instrument is the Challenger double energy $\mathrm{x}$-ray absorption instrument from France DMS Company, the applied software is the rat analysis software provided by DMS Company. Data of rat mean weights and BMD during experiment were shown in Tables $\mathbf{1}$ and $\mathbf{2}$.

\section{METHOD OF PARAMETER IDENTIFICATION FOR BONE GROWTH EQUATION}

\subsection{Bone Growth Equation}

"Stress and growth is the key problem in the study of Biomechanics [4]." The stress stimulus and the adaptability towards the stimulus are the basic rules of growth and remodeling of bone. Studying the relationship between growth and stress, we can understand the remodeling mechanism of bone from the point of foundation. Mathematical description of bone self-optimization process based on Wolff's law was as follows (as shown

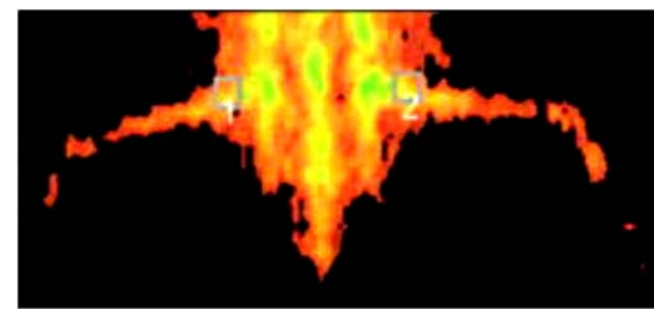

Figure 1. Measurement for bone mineral density.

Table 1. Data of rat mean weights during experiment (g).

\begin{tabular}{ccccc}
\hline & Week 1 & Week 2 & Week 3 & Weed 4 \\
\hline $\begin{array}{c}\text { Group } \\
\text { Experiment }\end{array}$ & $181.0 \pm 16.0$ & $186.2 \pm 17.9$ & $194.9 \pm 17.4$ & $200.8 \pm 18.0$ \\
$\begin{array}{c}\text { Group } \\
\text { Comparison }\end{array}$ & $209.1 \pm 19.0$ & $215.1 \pm 19.7$ & $220.6 \pm 20.0$ & $223.9 \pm 20.0$ \\
& Week 5 & Week 6 & Week 7 & Week 8 \\
$\begin{array}{c}\text { Group } \\
\text { Experiment } \\
\begin{array}{c}\text { Group } \\
\text { Comparison }\end{array}\end{array}$ & 211.5 $\pm 17.6 \pm 14.7$ & $188.9 \pm 15.6$ & $199.0 \pm 16.2$ & $204.5 \pm 16.4$ \\
\hline
\end{tabular}

Table 2. Data of mean BMD of rat proximal femur during experiment $\left(\mathrm{g} / \mathrm{cm}^{2}\right)$.

\begin{tabular}{lcccc}
\hline & Week 1 & Week 2 & Week 3 & Week 4 \\
\hline Group & $0.2378 \pm$ & $0.2584 \pm$ & $0.2746 \pm$ & $0.2902 \pm$ \\
Overloading & 0.0473 & 0.0362 & 0.0440 & 0.0418 \\
Group & $0.2508 \pm$ & $0.2584 \pm$ & $0.2618 \pm$ & $0.2649 \pm$ \\
unloading & 0.0476 & 0.0410 & 0.0471 & 0.0469 \\
Group & $0.2328 \pm$ & $0.2518 \pm$ & $0.2665 \pm$ & $0.2805 \pm$ \\
comparison 1 & 0.0333 & 0.0412 & 0.0485 & 0.0425 \\
Group & $0.2538 \pm$ & $0.2757 \pm$ & $0.2908 \pm$ & $0.3064 \pm$ \\
comparison 2 & 0.0294 & 0.0475 & 0.0517 & 0.0441 \\
& Week 5 & Week 6 & Week7 & Week 8 \\
Group & $0.3002 \pm$ & $0.3086 \pm$ & $0.3155 \pm$ & $0.3179 \pm$ \\
Overloading & 0.0600 & 0.0453 & 0.0512 & 0.0578 \\
Group & $0.2635 \pm$ & $0.2609 \pm$ & $0.2583 \pm$ & $0.2529 \pm$ \\
unloading & 0.0616 & 0.0472 & 0.0580 & 0.0599 \\
Group & $0.2888 \pm$ & $0.2941 \pm$ & $0.2977 \pm$ & $0.2980 \pm$ \\
comparison 1 & 0.0432 & 0.0575 & 0.0509 & 0.0484 \\
Group & $0.3155 \pm$ & $0.3214 \pm$ & $0.3249 \pm$ & $0.3248 \pm$ \\
comparison 2 & 0.0459 & 0.0552 & 0.0427 & 0.0430 \\
\hline
\end{tabular}

in Eq.1) [3].

$$
\frac{d \rho}{d t}=B(S-K)
$$

where $\rho$ is the apparent density of the trabecular bone structure; $t$ is time; $S$ represents mechanical stimulus, here it is equivalent stress; B represents the growth coefficient; $K$ represents a goal stress state. Among them, the selection and quantification of $\mathrm{B}$ and $K$ are the keys of establishing bone growth equation.

For the time interval was constant as 1 week, Eq.1 could be simplified as:

$$
\Delta \rho=B(S-K) \Delta t
$$

We defined:

$$
f=\min \sum_{i=1}^{n}\left(\Delta \rho^{2}-\Delta \rho_{\exp }{ }^{2}\right)
$$

Then, the object function for identifying $B$ and $K$ was generated.

\subsection{Parameter Identification and Analysis}

Lots of theories and Algorithms towards inversion have been studied [2,5]. Thus far, there are so many popular optimization algorithms such as ant group algorithm, genetic algorithm and analogue anneal algorithm. BFGS algorithm was explored by Broyden, Fletcher, Goldfarb and Shanno in 1970, which was a kind of variable metric method. In this paper, BFGS method was put forward to serve the inversion problem.

BFGS procedure was designed under Microsoft Visual Basic 6.0; it was debugged successfully under both Windows 2000 and Windows XP system. 
The BMD value of rat proximal femur obtained every week was categorized into four groups: left hindlimb of comparison group, right hindlimb of comparison group, left hindlimb of experiment group and right hindlimb of the experiment group. The variation quantity per week of BMD- $\triangle \mathrm{BMD}$ was regarded as $\mathrm{d} \rho / \mathrm{d} t$ and was input into program. The outer load $\mathrm{P}$ of each group was defined as body mass/4, body mass/4, body mass/ 3 and body mass/ googol, Cowin considered that the stimulus of bone growth equation was linear or quadric with strain in 1976 and 1981 respectively. Later, Huiskes [6] introduced stain energy density as the stimulus in 1987. According to Huiskes, we defined stimulus as p2/BMD2. The stimulus $\mathrm{S}$ and goal stress $\mathrm{K}$ are same in dimension, which unit is $\frac{m N^{2} * \mathrm{~cm}^{4}}{\mathrm{~g}^{2}}$, and the unit of growth coefficient $B$ is

$$
\frac{\Delta g * g^{2}}{\Delta W e e k * m N^{2} * m^{6}} \text {. }
$$

\section{CONCLUSIONS}

From the results (as shown in Figure 2), we can see that the bone growth coefficient $B$ diminished rapidly with time, the changing trend can be simulated with index function. The threshold value $K$ showed an ascend trend with time, it can be simulated with polynomial function (as shown in Figure 3). Inversion results must be proved by comparing with positive analysis [7], so these results of $B$ and $K$ together with weight of comparison group were substituted into bone growth equation. The obtained BMD-Time simulated curve was compared with the one of comparison group of the experiment in the other year (as shown in Figure 4).

The inversion results in this paper indicate that bone growth coefficient B grows small with time. At week 10, it approaches zero approximately, whereas factor $K$ increased remarkably at week $1,2,3,4$, and 5 , at the week $6,7,8,9$ and 10 , the increasing speed begins to decrease. 10 weeks later, it approaches a plane line. This illuminated that the rapid growing period of bone went to an

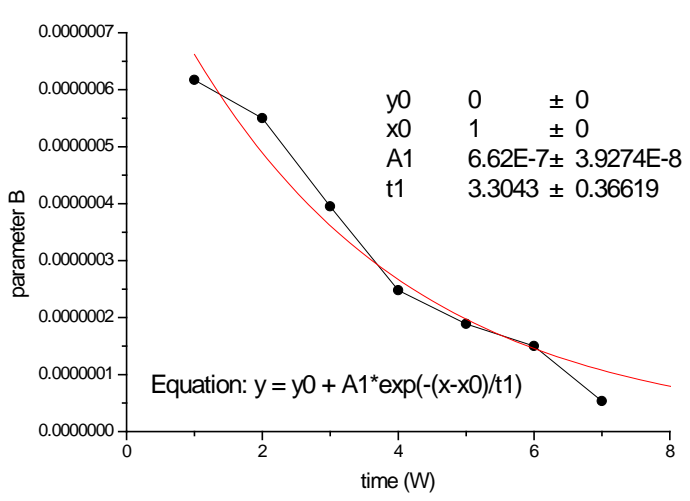

Figure 2. Result and growing tendency of $B$.

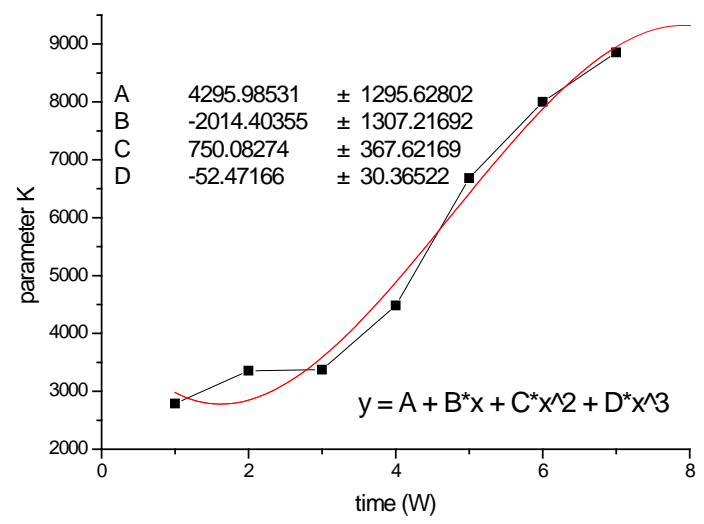

Figure 3. Result and variation tendency of $K$.

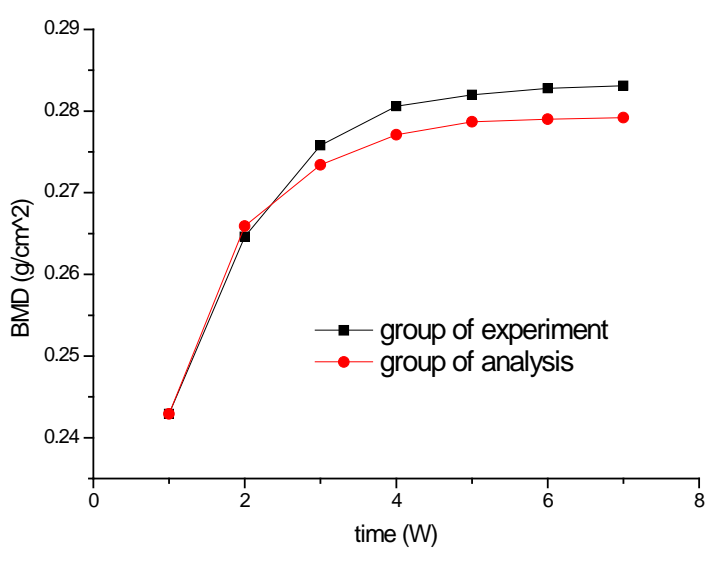

Figure 4. Comparison between analysis and experiment.

end and the bone entered a slow-growing stage. That is, bone formation and absorption had reached a balanced state and rat femur BMD had stopped rising basically.

The above inversion outcome is coincidence with the actual growth condition of rats (as shown if Figure 4) [8].

\section{DISCUSSIONS}

By now, human beings understand themselves far more than enough, studies on the causes of diseases, treatments and relationships between the human body and the surrounding environments are greatly confined for the lack of accurately-quantified model. In this paper, the unknown parameters in the bone growth equation by using inversion method based on animal experiments were provided, the relationships between environmental stress and bone growing in rats were also quantified. In addition, the bone growth coefficient $B$ was the function of the time as well as the space. $B$ and $K$ were usually regarded as constants [9] for easy calculation reason, but it's more reasonable to be regarded as functions of time because bone growth coefficient and stimulus threshold could vary during different growing periods. In clinics, parameters $B$ and $K$ play important roles and were closely 
related with treatments and disease recovery [10,11].

In this paper, some assumption and predigestion about the environmental stimulus were investigated. The shape of proximal femur is inalterable and BMD has uniform distribution; Overloading group, underloading group and comparison group were defined as equal parts of weight respectively, outer stimulus was defined as a function of weight and BMD. Actually, the anatomy of rat proximal femur was irregular and the stress distribution was complex. In order to get the data of objective and specific condition of stimulus, we had to work on numerical result by creating a three-dimensional model of bone based on CT images with the analysis of finite element method software. This will be left to further research. The emphasis of this study is the idea of applying parameter identification method to the quantification of bone growth equation. Though outer stimulus was simplified, but the result of both animal experiments and data analysis was acceptable compared with that of reference [8]. The differences may exist during the whole experiments, but the idea of creating numerical biological model by combining animal experiments and inversion theory was novel and it gave a perfect illustration of the creating of the self-adaptation model of human body.

Nowadays, the inversion method is being applied extensively in natural sciences, engineering and many other fields [6]. As known to all, in normal problems, we know the reasons to seek the results, whereas in inversion, we know the results to seek the reasons. The Human body is a complex system and there exists extensive coupled biomechanical phenomena, which is still largely immeasurable and unknown. Therefore, a new requirement was brought forward for modern medicine: by inversion method, namely according to some phenomena, measurement results and pre-enacted mathematical mode, we can deduce the unknown physical parameters and changing rule by reversed process, modify it by mathematical method and investigate it in depth. With the development of human society, life science is a new area that we must encounter. If chemistry has driven the development of life science in 20th century, the study of combining of biochemics and biomechanics based on the inversion and modern computing techniques will gain the achievements that exceed human's imagination. This study is one of the steps to create quantitative biomechanical model of vivo bone and it could also provide clues and references to establish biomechanical model of other organs or tissues in humans.

\section{REFERENCES}

[1] Feng, Y.Z. (1983) Biomechanics brief. Science Press, Beijing. (in Chinese)

[2] Wang, D.G. (2001) Nonlinear inversion algorithms and applications, doctoral dissertation of Dalian University of Technology. Dalian University of Technology Press, Dalian. (in Chinese)

[3] Wolff, J. (1884) Das Gesetz der transformation der inneren architectur der knochen bei pathologischen veränderungen der äusseren knochenform. Sitzg. Physik-Math, Klausuren, Sitz. Ber. Press, Berlin.

[4] Tao, Z.L. (1996) Relationship between growth and stressone prospect of biomechanics, advances of biomechanics. 5th Science Congress of Biomechanics, Chengdu University of Technique Press, Chengdu, 15-17. (in Chinese)

[5] Wang, D.G., Liu, Y.X. and Li, S.J. (2003) Summaries of elastic mechanics inversion method. Advances of $\mathrm{Me}$ chanics, 2, 166-174. (in Chinese)

[6] Huiskes, R., Weinans, H.J., Grootenboer, H.J., et al. (1987) Adaptive bone remodeling rheory applied to prostheticdesign analysis. Biomechanics, 20, 1135. http://dx.doi.org/10.1016/0021-9290(87)90030-3

[7] Chinese Academy of Sciences (2000) Report on science development. Science Press, Beijing. (in Chinese)

[8] Danielsen, C.C. (1993) Cortical bone mass, composition, and mechanical properties in female rats in relation to age, long-term ovariectomy, and estrogen substitution. Calcified Tissue International, 52, 26. http://dx.doi.org/10.1007/BF00675623

[9] Zhu, X.H., He, G., Zhu, D., et al. (2002) A study of the effect of non-linearities in the equation of bone remodeling. Journal of Biomechanics, 7, 951-960 http://dx.doi.org/10.1016/S0021-9290(02)00028-3

[10] Zhao, W.Z., Liu, Y.X., Zhang, J., et al. (2003) Biomechanical analysis of internal implant failure after internal fixation of compressing steel plate. Journal of Medical Biomechanics, 18, 50-53. (in Chinese)

[11] Lin, Y.J., Yang, J.W., Chen, J., et al. (2000) Kinesiatrics for osteoporosis. Physical Medicine and Rehabilitation of Foreign Medicine, 20, 97-101. (in Chinese) 